\title{
Pharmacy Automation and Technology
}

\section{Are You Practicing in a Learning Health System?}

\author{
Bill G. Felkey, MS, " and Brent I. Fox, PharmD, PhD
}

\begin{abstract}
Learning never ceases. While continuing education is important for all of us, we are not speaking of licensure requirements. We are speaking of learning within your health system that systematically incorporates knowledge gained through research and clinical care into individual care.
\end{abstract}

A $s$ professors, we try to equip our students with the knowledge and technology that will enable them to be more effective and efficient. We hope that they will act as change agents in their new practice settings. Instead, we often see recent graduates conforming to what is going on as the current standard of practice within 1 month of employment, even though they have skills and knowledge of other approaches to share. This is a missed opportunity, especially in this time of significant changes in health care.

Many of these changes require that health care systems modify the way they offer their services. Dropping fee-for-service reimbursement models and replacing them with population health is one of these changes. Nearly every health system is straddling the old payment systems (with the inherent incentives) and preparing for population health (with entirely different incentives). In a previous column we explored the potential of and challenges associated with precision medicine, some of which are technical while others are workflow in nature. Also, Meaningful Use continues to be an important driver in our hospitals and health systems. Specifically, patient engagement has gained increasing importance in the form of patients accessing and managing (to an extent) their health information.

Add the political fallout of the recent presidential election where the currently (at the time of this writing) proposed 100-day agendas include repealing the Affordable Care Act and replacing it with a new or modified system. Our country may not be able to politically justify the cost of health care continuing to exceed $17 \%$ of the GDP, and we know that the government must get a handle on health care for a number of reasons. It's time that we all buckle our seatbelts in anticipation of new and challenging changes in the health care field.

With all this potential change, it is important to ask whether your health system is a scientifically and ethically learning health care system. We're talking more than just-in-time decision support technology. We're talking more than satisfying continuing education requirements. At its foundation, does your health system promote and live through a learning perspective mode in its day-to-day operations?

To answer "yes" to this question, your leadership must actively be attempting to instill a culture of learning using supportive system competencies. These supportive systems must supply the right data to the right person at the right time. Ideally, multidisciplinary teams will modify this basic tenant to include the right provider providing the right care in the right facility to the right patient at the right time.

In addition, the institution must have a system to aggregate and normalize data from each of the care teams' native applications to formulate a single source of truth at each care decision point. This requires analytics and tremendous interoperability that becomes integrated at the point of care throughout the help system continuum. We are currently evaluating systems that are attempting to bring this much-needed support into reality.

The most progressive health institution using the best systems will fail if patients are not fully engaged and empowered to participate in their own health. For example, the former Surgeon General C. Everett Koop said, "Drugs don't work in patients who don't take them." Other patients would rather smoke, drink, or eat as they please than live. Patient failures happen

"Professor Emeritus, Auburn University, Auburn, Alabama; ${ }^{\dagger}$ Associate Professor, Department of Health Outcomes Research and Policy, Harrison School of Pharmacy, Auburn University, Auburn, Alabama 
most frequently when patients don't know what to do, don't know how to do it, or are not motivated for their self-care management. There are many human and technological systems that address this challenge.

The last area that a learning health system must have in place is the alignment of incentives. When the system is based on fee-for-service, full waiting rooms and even fuller procedure scheduling is the only way providers can pay the bills and live at the lifestyle level they desire. When population health makes the filling of acute care beds something to avoid, then we are confident that the new "right" way to do things will quickly change. Remember that the culture change in your health system can either be top-down or bottom-up. If you answered that your health system is not a learning place in the way you feel that is needed to cope with all of the changes taking place, we would like to encourage and support your advocacy. You can reach Bill at felkebg@auburn.edu or Brent at foxbren@auburn.edu to continue the conversation. 
